\title{
Allergen immunotherapy
}

\section{SUMMARY}

Allergen immunotherapy reduces the symptoms of allergic disease by inducing tolerance to specific allergens. It can be given sublingually or by subcutaneous injection.

Immunotherapy is the only form of treatment which modifies abnormal immune reactivity to a specific allergen, rather than simply suppressing symptoms. It may alter the natural history of atopic disease.

Allergen immunotherapy is effective for respiratory allergy (rhinitis and asthma) and venom allergy such as bee stings. Currently immunotherapy has no role in the routine management of food allergy, but research is ongoing.

\section{Introduction}

Allergen immunotherapy was first reported in the successful treatment of pollen-induced allergic rhinitis in 1911. It reduces the patient's abnormal immunoreactivity to harmless environmental antigens (allergens). The repeated administration of the allergen induces tolerance. A reduction of allergeninduced symptoms should occur within months. After a 3-5 year maintenance period, this benefit should be long-lasting if not permanent.

For most of its history immunotherapy has been given by subcutaneous injection. As injecting an allergen has the potential to cause anaphylaxis, there is an initial cautious 'updosing' phase followed by a prolonged maintenance phase with regular injections. Recently, sublingual administration of allergen has been found to be effective in allergic respiratory disease.

There is evidence from meta-analyses for the efficacy of subcutaneous and sublingual immunotherapy in the management of allergic rhinitis and asthma. ${ }^{1-3}$ Subcutaneous immunotherapy is also effective for venom allergy. ${ }^{4}$ The relative efficacy of sublingual and subcutaneous immunotherapy in respiratory allergy remains unclear.

\section{Mechanisms of action}

Several mechanisms are proposed to contribute to the effect of allergen immunotherapy. They include the induction of IgG 'blocking' antibodies (which inhibit binding of allergen to $\lg \mathrm{E}$ ), T cell anergy, switching of T-helper 2 (allergic phenotype) cells to T-helper 1 , and the induction of regulatory $T$ cells which suppress the immune response. However, even in clinically successful immunotherapy, allergen-specific IgE is still present and allergy tests usually remain positive.

\section{Management of allergic diseases}

Management of allergen-induced respiratory symptoms starts with the identification of relevant allergens and avoiding them where possible. Oral or topical antihistamines and intranasal corticosteroids can be effective regardless of the specific allergic cause. Immunotherapy is indicated when the patient's symptoms are moderate to severe, symptoms interfere with function or quality of life, avoidance of the allergen is difficult or impossible, and other treatments are unsatisfactory.

It is important to ensure that the allergens used for immunotherapy are the ones that are causing the patient's symptoms. This is established by a history of symptom patterns matching allergen exposure and confirmed by skin prick tests or serum allergenspecific lgE tests.

Immunotherapy for asthma is problematic because asthma is usually multifactorial. Moderate to severe asthma is also a risk factor for adverse reactions to immunotherapy. However, there is evidence that subcutaneous immunotherapy improves bronchial hyperreactivity and reduces symptoms and medicine use for asthma. ${ }^{3}$

In patients who have had anaphylaxis from an insect sting, immunotherapy is highly effective in reducing the risk of reactions to subsequent stings. In Australia, anaphylaxis most commonly occurs from bee stings, but can also be caused by stings from native and imported wasps and Jack-Jumper ants.

\section{Allergens for immunotherapy}

Allergens are manufactured for both diagnosis and immunotherapy. They are purified protein extracts from allergenic substances. Diagnostic extracts should be from a single species only and contain all relevant
William B Smith

Senior consultant Department of Clinical Immunology and Allergy Royal Adelaide Hospital Adelaide

\section{Key words}

allergic rhinitis, anaphylaxis, asthma, venom

Aust Prescr 2014;37:87-9 


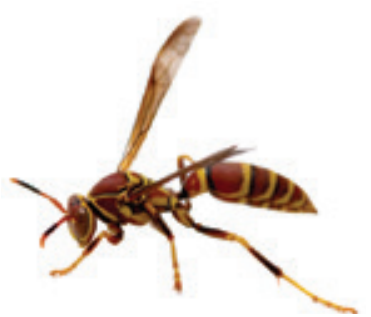

Paper wasp

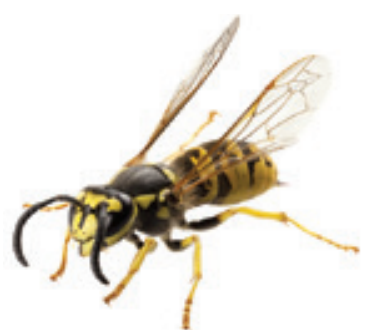

European Wasp

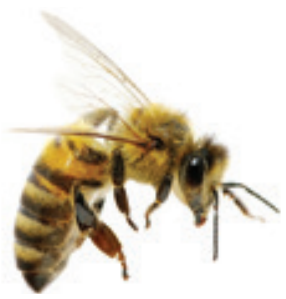

Honeybee

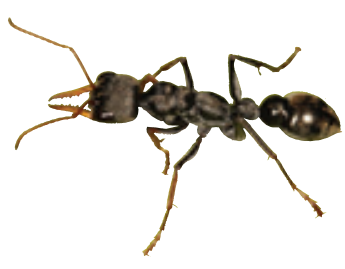

Jack-Jumper ant allergenic proteins. However, allergen extracts are poorly defined pharmacologically and biochemically and poorly standardised. Extracts from different manufacturers vary considerably and this could influence the effectiveness of diagnosis and treatment. Recombinant allergenic proteins are starting to be used in diagnosis, but not yet for therapy.

Common allergen classes in respiratory disease are pollens (grass, weed and tree), dust mites, moulds and animal danders. Pollens tend to cause seasonal rhinitis although seasonal patterns vary with pollen type and region. Other allergens may cause perennial rhinitis, depending on exposure patterns. Allergens from each of these groups are available for immunotherapy.

The allergens used for immunotherapy may be single extracts from an individual species or mixtures of different allergen species. Mixtures of different allergen classes may not be compatible. ${ }^{5}$ The patient's sensitisation pattern and major exposures guide the selection of allergens. Many patients are sensitised to multiple allergens either within one of the main classes (for example pollens) or from several classes. This may be due to cross-reactive allergy or due to separate independent sensitisations. It is unclear whether all relevant allergens need to be included in the mixture for optimal results (some mixtures contain potentially suboptimal amounts of each allergen) or whether a small number of key or dominant allergens will suffice.

\section{Treatment}

Allergen immunotherapy is usually prescribed by physicians or paediatricians who have received subspecialty training in clinical immunology and allergy. This is to ensure optimal patient and allergen selection and to manage the risks of immunotherapy. Usually therapy begins with weekly injections in an outpatient or clinic setting. Venom immunotherapy may be introduced with a rapid-updosing 'rush' protocol over 2-5 days. This is done in a hospital day-patient setting because of the increased risk of reactions. Most injections can be given by specialists or GPs. Free e-training in immunotherapy is available from the Australasian Society of Clinical Immunology and Allergy. ${ }^{6}$ Practitioners need the skill and equipment to be able to manage anaphylaxis (see Australian Prescriber wallchart ${ }^{7}$ ).

\section{Subcutaneous immunotherapy}

There are two main ranges of injectable immunotherapy products in Australia:

- an aluminium hydroxide conjugated formula which may be ordered in standard preparations or in individual mixtures

- an aqueous formulation usually prepared by the allergy specialist.
Some allergens are registered therapeutic goods whereas conjugated allergen mixes are available on a named-patient basis. Only certain venom allergens are available on the Pharmaceutical Benefits Scheme. These are for bee, European (Vespula) and paper wasp (Polistes). Jack-Jumper ant venom immunotherapy is available in some centres (Royal Adelaide Hospital and Royal Hobart Hospital) at the patient's expense.

Subcutaneous immunotherapy is usually effective for symptom reduction. The responses may be partial rather than total, so it should not be assumed that other treatments and avoidance strategies will no longer be needed. In addition, there is a subgroup of patients who do not improve with immunotherapy. This may be because a suboptimal allergen was used or the symptoms were actually caused by nonallergic disease (for example, chronic rhinosinusitis). Sometimes immunotherapy fails for unknown reasons.

\section{Injection technique}

Injections of allergen are administered subcutaneously, usually in the posterior part of the upper arm, using a fine gauge needle (26/27G) and $1 \mathrm{~mL}$ syringe (insulin syringes are ideal). A complete detailed guide to the administration of subcutaneous immunotherapy injections is available at www.allergy.org.au/healthprofessionals/papers/scit-treatment-plan.

\section{Adverse effects}

Subcutaneous immunotherapy carries risks which include immediate reactions such as anaphylaxis, and delayed reactions such as local swelling and more rarely, exacerbations of asthma or atopic eczema. The risk of immediate reactions can be reduced by premedication with antihistamines, but medical observation for 30-45 minutes after each injection is mandatory, including during the maintenance phase of treatment even if the injections have previously been well tolerated.

\section{Sublingual immunotherapy}

Alternative routes of delivery for immunotherapy have been sought to improve safety and ease of use while retaining effectiveness. Sublingual immunotherapy emerged in the mainstream literature in the 1990s. There is now evidence ${ }^{2}$ for the efficacy of sublingually administered allergens in respiratory allergy. Higher doses of allergen are required compared with subcutaneous immunotherapy. The allergen is absorbed through the oral mucosa and studies have shown systemic immunological changes similar to those of subcutaneous immunotherapy. The majority of evidence relates to single allergens or oligoallergen mixtures, mainly pollen and mite, in allergic rhinitis, but evidence also exists for efficacy in asthma. 
Sublingual immunotherapy is available in tablets or in drop form (pump bottles or plastic ampoules). Liquid drops for sublingual immunotherapy are available to order on a named-patient basis as single allergens or allergen mixtures. The only sublingual immunotherapy tablet currently registered is a fixed composition mixture of five pollens from the rye grass family.

Sublingual immunotherapy is convenient and can be administered at home. Various protocols are currently suggested, although the default is daily treatment for three years (the same total term as subcutaneous immunotherapy). Alternate daily schedules and pre/co-seasonal-only schedules are also used. While the acceptability of sublingual immunotherapy is high, adherence to the full long-term program is poor. ${ }^{8}$

\section{Adverse effects}

Sublingual immunotherapy has a substantial safety advantage over subcutaneous injections. It has mostly transient local adverse effects with very few reports of systemic reactions. ${ }^{9}$

\section{Future developments}

An important finding in both subcutaneous and sublingual immunotherapy is the potential for altering the natural history of atopy. There is evidence for a reduction in the new onset of asthma ${ }^{10}$ in those treated for allergic rhinitis and also a reduction in the incidence of new allergic sensitisations." These findings are intriguing and promising, but require further replication. If confirmed, they suggest that immunotherapy may reduce the overall burden of allergic disease and should be used earlier in allergic respiratory disease, not just when other treatments have failed.
Recombinant allergens or modified (peptide) allergens may provide advantages and are currently in development. Peptides and modified proteins may be constructed in such a way as to avoid IgE-binding. This reduces the risk of anaphylaxis while retaining the $T$ cell epitopes which induce regulatory $T$ cells to suppress allergy.

Immunotherapy for food allergy is not routinely practised and hitherto was considered too risky. However, there has been a great deal of recent investigation into inducing specific oral tolerance for egg, milk and nut allergy. This involves gradual introduction of the allergenic food under carefully controlled conditions. It is still considered a researchonly procedure. Concerns remain regarding the considerable risk of acute allergic reactions, the induction of eosinophilic enteritis, and whether permanent tolerance (as opposed to temporary desensitisation) can be achieved. $<$

\section{Conclusion}

Allergen immunotherapy is an important modality in the management of respiratory allergic disease and venom allergy. Immunotherapy for respiratory allergic disease has been expanded from the traditional injection method to include sublingual administration which has near-equivalent efficacy. $<$

William Smith has attended an interstate presentation sponsored by Stallergenes, and while at a conference in London, attended a sponsored visit to the Stallergenes factory in Paris. Stallergenes are manufacturers of allergen immunotherapy products.
SELF-TEST QUESTIONS

True or false?

3. Anaphylaxis can occur during the maintenance phase of injection immunotherapy.

4. Severe asthma increases the risk of an adverse reaction to immunotherapy.

Answers on page 107

\section{REFERENCES}

1. Calderon MA, Alves B, Jacobson M, Hurwitz B, Sheikh A, Durham S. Allergen injection immunotherapy for seasonal allergic rhinitis. Cochrane Database Syst Rev 2007. CD001936.

2. Radulovic S, Calderon MA, Wilson D, Durham S. Sublingua immunotherapy for allergic rhinitis. Cochrane Database Syst Rev 2010. CD002893.

3. Abramson MJ, Puy RM, Weiner JM. Injection allergen immunotherapy for asthma. Cochrane Database Syst Rev 2010. CD001186.

4. Boyle RJ, Elremeli M, Hockenhull J, Cherry MG, Bulsara MK Daniels $M$, et al. Venom immunotherapy for preventing allergic reactions to insect stings. Cochrane Database Syst Rev 2012. CD008838.

5. Grier TJ, LeFevre DM, Duncan EA, Esch RE, Coyne TC Allergen stabilities and compatibilities in mixtures of highprotease fungal and insect extracts. Ann Allergy Asthma Immunol 2012;108:439-47.

6. Australasian Society of Clinical Immunology and Allergy. Health Professionals. Health Professionals e-training. 2012. www.allergy.org.au [cited 2014 Apr 17]

7. Anaphylaxis: Emergency management for health professionals [wall chart]. 2011. Aust Prescr 2011;34:124.

8. Savi E, Peveri S, Senna G, Passalacqua G. Causes of SLIT discontinuation and strategies to improve the adherence: a pragmatic approach. Allergy 2013;68:1193-5.
9. Canonica GW, Bousquet J, Casale T, Lockey RF, Baena-Cagnani CE, Pawankar R, et al. Sub-lingual immunotherapy: World Allergy Organization Position Paper 2009. Allergy 2009;64 (Suppl 91):1-59.

10. Möller C, Dreborg S, Ferdousi HA, Halken S, Høst A, Jacobsen $\mathrm{L}$, et al. Pollen immunotherapy reduces the development of asthma in children with seasonal rhinoconjunctivitis (the PAT-study). J Allergy Clin Immunol 2002;109:251

11. Pajno GB, Barberio G, De Luca F, Morabito L, Parmiani S. Prevention of new sensitizations in asthmatic children monosensitized to house dust mite by specific immunotherapy. A six-year follow-up study. Clin Exp Allergy 2001;31:1392-7.

Image credits

Paper Wasp (polistes dorsalis): Melinda Fawver/shutterstock.com Wasp Vespula germanica species: paulrommer/shutterstock.com Bee: irin-k/shutterstock.com

Jumping-jack ant: Katarina Christenson/shutterstock.com 